\title{
COBALT CHROMIUM DENTURE DESIGNS IN GENERAL PRACTICE
}

W.A. Mahmood, M.F. Mohd. Sidek. Cobalt Chromium Denture Designs in General Practice. Annal Dent Univ Malaya 2001; 8: 29-34.

\section{ABSTRACT}

This is a preliminary survey on cast partial denture designs in the commercial dental laboratories. This survey was carried out for a month in three commercial dental laboratories in Klang Valley and Shah Alam which produce metal dentures in cobalt-chromium. One hundred and ten questionnaires with the designs were collected and analysed. The framework design on the cast was transferred into the design sheet section of the questionnaire.

The aim was to investigate communication regarding denture design between clinicians and dental technician and the dentists' dependency on the technician. The design of cobalt-chromium partial dentures in relation to oral health was also assessed.

The results indicated that $43.6 \%$ of the dentist who used the three laboratories delegated their removable partial design work to the dental technician. More than half of the dentists had some communication with the technicians, and only $18.2 \%$ of the dentists prescribed clear instructions with details of components regarding denture design. Continuing dental education on partial denture design for both clinicians and dental technicians would be of value to provide reinforcement in the knowledge of the basic concept on denture designing. Communication and understanding between both parties would probably improve the quality of cobalt-chromium dentures constructed.

Key words: Removable partial denture, cobalt-chromium partial dentures, partial denture designs

\section{INTRODUCTION}

Removable partial denture (RPD) design should be based on sound principles following detailed clinical examination. Denture design for each individual patient should be based on the state of the remaining teeth and the status of his oral health. Where denture casting work is concerned, there should be more emphasis on preliminary work such as model surveying. Extra attention is required for precision and accuracy due to the casting process as compared to the acrylic partial denture.

The Academy of Prosthodontics (1) stated that treatment planning, mouth preparation and designing removable partial denture are the dentists' professional responsibilities and this is supported by other workers from previous studies (2-5). These are clear indications that dentists should accept full responsibility in designing
Original Article

1W. A. Mahmood, 2M. F. Mohd. Sidek
I Lecturer
Department of Prosthetics
Faculty of Dentistry
University of Malaya
50603 Kuala Lumpur
2 Year V undergraduate student (2000/2001)
Faculty of Dentistry
University of Malaya
50603 Kuala Lumpur

partial dentures, because they have acquired the necessary biological knowledge to appreciate the various factors concerned with the partial denture design.

Surveys on partial denture designs and dental laboratories have been carried out in the United Kingdom, Sweden as well as in the United States and Canada (2-14). Despite having basic undergraduate training, dentists still rely upon technicians to design partial dentures especially cobalt-chromium partial dentures (5-7,9-14). Von Steyern et al (6) reviewed the communication between dentist and technician and their role in designing partial dentures (7-11). The review showed that dentists played a minor role in designing partial dentures. The dentist did not provide adequate prescription on the denture design to the dental technician. This was found to be a common practice in Sweden, United Kingdom, United States and Canada (56,11-12,14).

The reasons for delegating denture design to the dental technicians were investigated in a study by Holt et al (14). They summarised the reasons as lack of confidence in producing designs by the dentist and insufficient exposure to clinical practice.

No survey of this kind has been done before in Malaysia. Therefore it is not known whether the same situation prevails here. Hence this preliminary study was initiated and was based on the work of Basker et al. $(10,12)$

The objectives of the study were to assess:

1) communication between dentist and dental technician with regards to removable partial denture design.

2) the dentists' dependency on the technician in the commercial laboratory with regards to removable partial denture design.

3) the dentists or the technician's ability to design cast partial denture in relation to sound principles, function and oral health. 


\section{MATERIAL AND METHODS}

Three dental laboratories which provide the facilities in cobalt chromium framework casting were identified. Each of these laboratories were informed of the purpose of the study. A set of questionnaire was used to collect the relevant information. This questionnaire was based on the work of Basker and Davenport (10) in 1978.

The three laboratories were visited for the period of a month. The number of casts examined depended on the availability of work sent at the time sf the study. All the cast framework designs were transferred on the design sheet section in the questionnaires. Details in terms of denture classification and other partial denture components were analysed. Each questionnaire was checked and placed in one of four categories:

1. Detailed - clear instructions with type of components prescribed by dentists together with details of position of clasps and occlusal rests.

2. Guided - insufficient information and requiring the technicians to make the final decision.

3. None - No prescription

4. Specific request - request for laboratory staff to design.

The Kennedy classification (15) was used in this study to classify the partially edentulous situations.

\section{RESULTS}

The information collected from this study was based on 110 cases available at the time of the study. The duration for data collection was confined to a month. The number of upper and lower casts studied are shown in Table 1.

The number of cobalt chromium framework produced by the 3 laboratories is described in Table 2 .

The majority of upper dentures were made up of bounded saddles (Kennedy Class III-48.57\%) while the

Table 1. Distribution of upper \& lower cast

\begin{tabular}{ccc}
\hline Cast & $\mathrm{n}=110$ & $\%$ \\
\hline Upper & 70 & 63.6 \\
Lower & 40 & 36.4 \\
\hline
\end{tabular}

Table 2. The number of Cobalt-Chromium frameworks produced by the laboratories studied

\begin{tabular}{cccc}
\hline & \multicolumn{2}{c}{ Cast } & \\
\cline { 2 - 3 } Laboratory & Upper & Lower & \\
\hline A & 28 & 17 & 45 \\
B & 13 & 8 & 21 \\
C & 29 & 15 & 44 \\
\hline
\end{tabular}

majority of the lower dentures were made up of free end saddle situations/casts (Kennedy Class I-40\%) as shown in Table 3.

Figure 1 showed that $43.6 \%$ of dentists did not prescribe any design instruction to the technician. Only $18.2 \%$ of the dentists wrote detailed instructions on the type of components used and the position of clasps and occlusal rests.

The type of support for each class of the framework is detailed in Figure 2 and 3 for the maxilla and mandible respectively.

The following analysis emphasise on designs related to the lower free end saddle situations. A summary of the types of connectors used is shown in Table 4. Figure 4 represents the application of the Rests, Proximal plate and 1-bar (RPI) system in the lower bilateral free end saddle situation. The designs of rests, clasps on lower casts and on premolar abutment teeth are represented in figures $5,6 \mathrm{a}$ and $6 \mathrm{~b}$.

\section{DISCUSSION}

The objective of partial denture design is not only to preserve the health and relationship of the remaining teeth but also the health of the oral tissue.

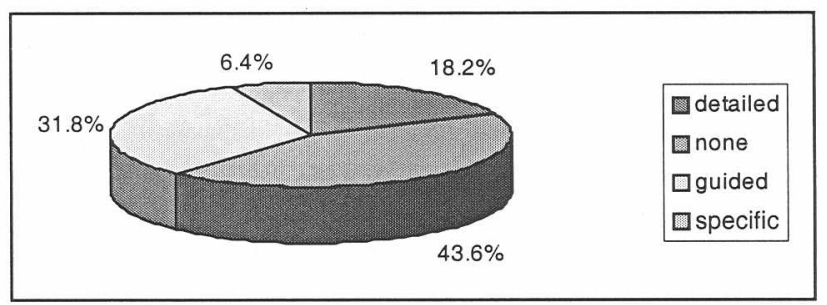

Figure 1. Communication between dentist and technician.

1. Detailed

- clear instruction with type of components prescribed by dentists together with details of position of clasps and occlusal rests.

2. Guided - insufficient information and requiring the technicians to make the final decision.

3. None - No prescription

4. Specific request - request for laboratory staff to design

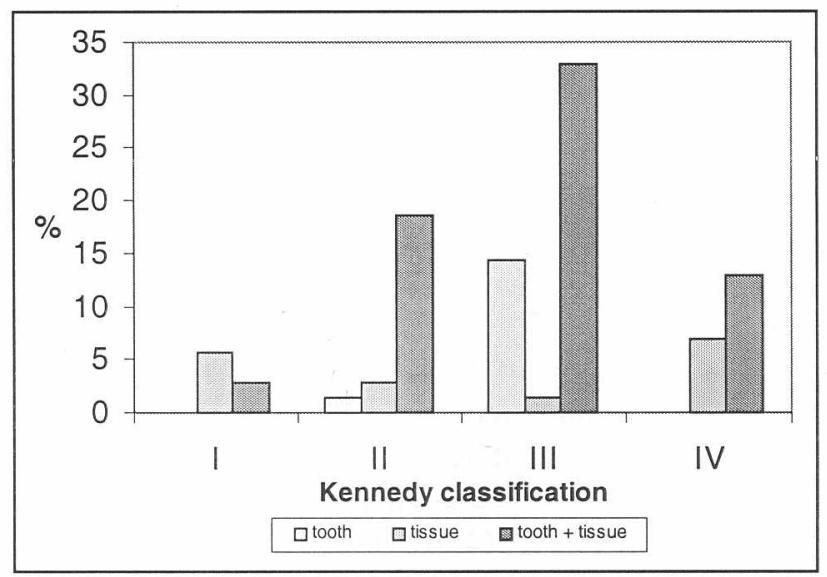

Figure 2. Type of support for each class of Cobalt-Chromium denture in the upper jaw (maxilla). 


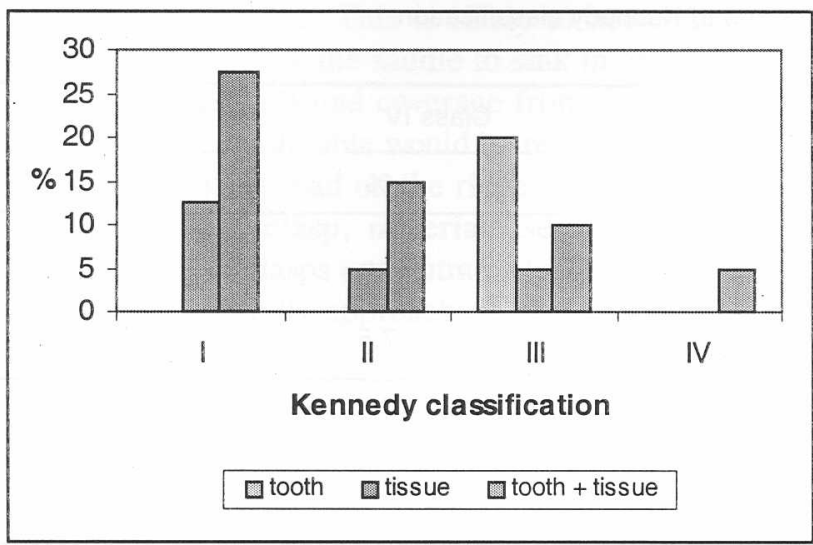

Figure 3. Type of support for each class of Cobalt-Chromium denture in the lower jaw (mandible).

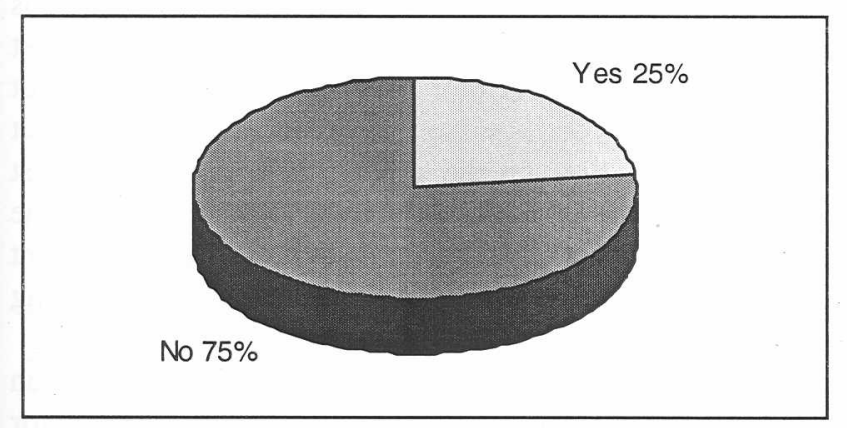

Figure 4. Application of RPI system in the lower bilateral free-end saddle situation.

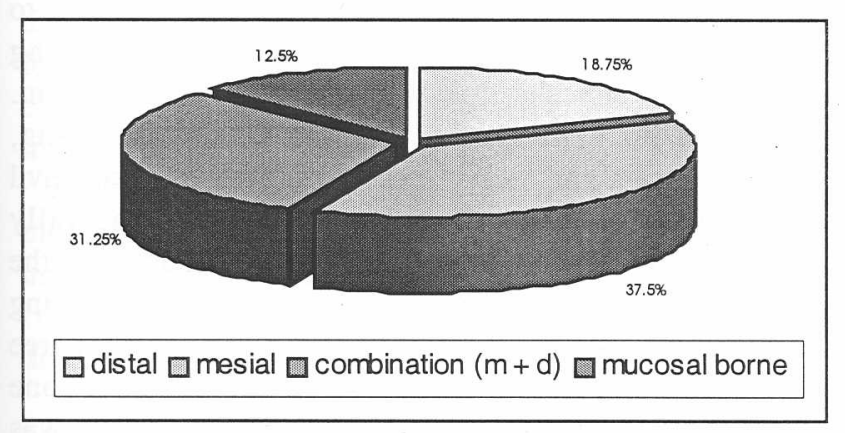

Figure 5. Rest placement on the most distal abutment (premolar) on the lower bilateral free-end saddle situation.

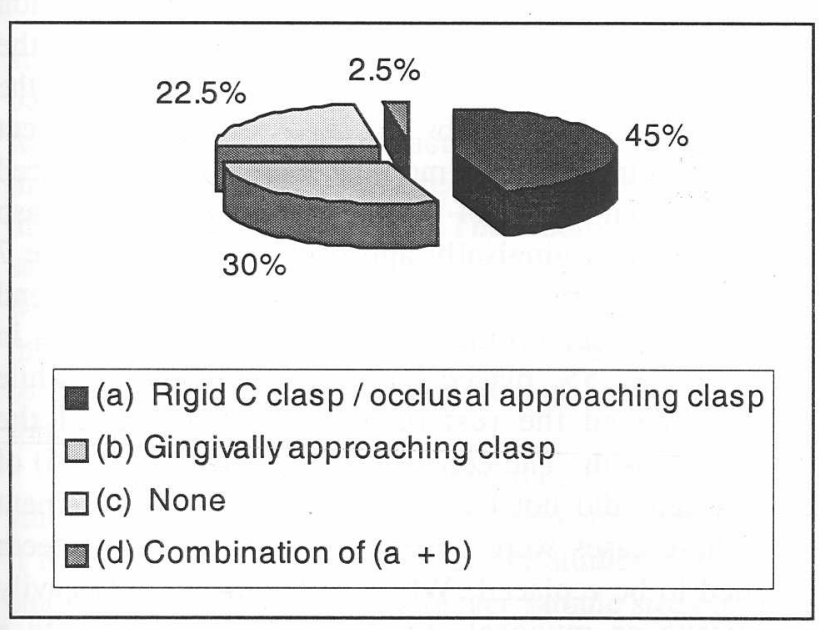

Figure 6a. Types of clasps used on the lower cast framework.

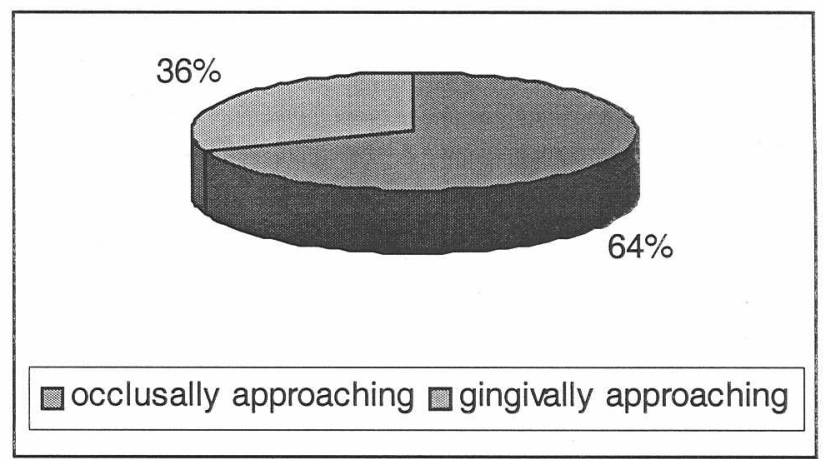

Figure $6 \mathrm{~b}$. Types of clasps used on the premolar abutments.

Dentists should be fully responsible for the partial denture design as they do the preliminary examination, oral diagnosis and the treatment planning. The status of the remaining teeth and tissues will influence the design. An ideal partial denture based on sound principles of partial denture design should contribute to total oral health care.

This preliminary study looked into the collaboration between dentist and technician in the outcome of cobalt chromium partial denture designs. The findings indicated that in the Klang Valley and Shah Alam, the pattern of dependency of dentist towards dental technician with regards to partial denture design is similar to the findings of workers from other parts of the world $(6-7,9-11,13)$. Figure 2 showed that $43.6 \%$ of dentists relied solely on technicians to design the dentures. Only $18.2 \%$ actually prescribed a detailed instruction to the laboratory technician. About $31.8 \%$ gave some guided instructions and a small percentage of $6.4 \%$ made specific requests for the laboratories to design the dentures.

The results suggested that nearly half of the dentists who used the laboratories delegated the design of the removable partial dentures to the technicians. About $50 \%$ of the frameworks were designed solely by the technicians. The main criteria was to check if the denture design conforms to the principle of maintaining oral health (16). Almost all designs except for a few provided less unnecessary tissue coverage and a wider spread of tooth tissue support. The frameworks showed that each Kennedy classification had a similar design. This may imply that the technicians use a common design for a specific class of partially edentulous situation.

In common with findings of previous workers $(10,12)$ there were more upper dentures constructed as compared to lower dentures. The ratio being 7:4 is shown in Table 1.

Basker et al (12) reported that more of the upper than lower dentures were requested. They claimed the reason was for aesthetics as would be the case with upper dentures. However, our study showed a different outcome. In the upper, there were more bounded saddle cases (Kennedy Class III) followed by Kennedy Class II and IV cases (Table 3) sent to the three laboratories. This indicated that there were less cases with missing posterior teeth and also anterior teeth in the cases studied. 
Table 3. Total number of dentures according to Kennedy classification

\begin{tabular}{|c|c|c|c|c|c|c|c|c|c|c|}
\hline \multirow[t]{2}{*}{ Cast } & \multicolumn{2}{|c|}{ class I } & \multicolumn{2}{|c|}{ class II } & \multicolumn{2}{|c|}{ class III } & \multicolumn{2}{|c|}{ Class IV } & \multicolumn{2}{|c|}{ Total $n=110$} \\
\hline & $\mathrm{n}$ & $\%$ & $\mathrm{n}$ & $\%$ & $\mathrm{n}$ & $\%$ & $n$ & $\%$ & $n$ & $\%$ \\
\hline Upper & 6 & 8.57 & 16 & 22.86 & 34 & 48.57 & 14 & 20 & 70 & 63.6 \\
\hline Lower & 16 & 40 & 8 & 20 & 13 & 32.5 & 3 & 7.5 & 40 & 36.4 \\
\hline
\end{tabular}

Table 4. Major connector used in the lower free-end situation

\begin{tabular}{lcc}
\hline Types of connector used & $\mathrm{n}=16$ & $\%$ \\
\hline Lingual Plate & 3 & 18.8 \\
Lingual Bar & 13 & 81.2 \\
\hline
\end{tabular}

Forty lower casts $(36.4 \%)$ were sent for framework casting (Table 3). There were slightly more Kennedy Class I cases (40\%) compared to $32.5 \%$ of cases with bounded saddle (Kennedy Class III). This may imply that when there is loss of posterior teeth, the need to masticate necessitated the patient to seek treatment. Other reasons for the loss in the posterior teeth would likely be extractions due to advanced periodontitis or carious lesions. Unrestored posterior region in the long term could develop into a chronic temporomandibular joint dysfunction and hence partial dentures would still be an invaluable treatment.

The situation in the lower arch is critical in providing effective posterior chewing table without causing undue stress to the denture bearing area of the residual ridge. The spread of the distribution of support provided by the designs are illustrated separately for upper and lower cast frameworks (Figures 2 \& 3 respectively).

In the upper casts (Figure 2), since there were more bounded saddles (Class III Kennedy), the type of support observed were derived from both tooth and tissue support. Only a few cases were observed to utilize tissue support. They were mainly involving class IV Kennedy (7\%) and class I Kennedy (5.7\%) situations (Figure 2). The designs analysed from the data indicated that broad palatal connectors were used when replacing more than 6 teeth. This was a sensible approach as the wide area of the palate was fully utilized for support with adequate rigidity provided by the palatal connectors while keeping clear of gingival margins.

In the lower denture designs (Figure 3), a similar observation of tooth and tissue support in Class I Kennedy situations was observed. The only difference was that for class III Kennedy situations, which was the second most frequently observed, the support derived was mainly from the tooth. On the whole, greater use of tooth support is encouraged in denture designing since the best load bearing structures are sound natural teeth and wherever possible, dentures should be totally tooth supported. Tooth support is provided by means of rests. However, although tooth support was used in the design, no tooth preparations for rest seats were made. Out of $18.2 \%$ (Figure 1) who prescribed the denture designs in detail, only 4 casts showed evidence of rest seat preparations. It was either that the rest seats were not prepared at all or they were done inefficiently so that they did not show on the model. It is hoped that rest preparation was deemed unnecessary by the dentist due to the clinical situation such as unopposed abutment teeth or there was sufficient clearance from the opposing upper teeth.

Denture construction for free end saddle situation in the mandible is the most challenging experience for dentists. This is due to the difference in the displacement of the teeth and mucosa in the free end saddle situation. The abutment teeth adjacent to the free end saddle will be subjected to forces transmitted from the denture to them via clasps and rests. The least damaging combination of occlusal rest and clasp is the RPI system. This is a resilient system utilising flexible clasping, direct retainers and mesial rests introduced by Kratochvil (17). He advocated an I- bar design of gingivally approaching clasp which has only point contact with the tooth and which is claimed to exert no adverse tipping force on the abutment tooth. From the 16 cases of free end saddle situations identified (Table 3), only one quarter utilised the RPI system (Figure 4). It was interesting to note that 2 of these cases were designed by the technicians.

Movement of the denture base towards the tissues in function is inevitable in the free end saddle situation due to the differences in the displaceability of the mucosa (18). The movement at the proximal end of the saddle can be prevented by placing a rest on the adjacent tooth. Loading would be more favourable if rest is placed on the mesial aspect of the tooth with a flexible clasp design such as gingivally approaching clasp. Figure 7 showed the distribution of rest placement in the free end saddle situation. From 16 cases of free end saddles in the lower $37.5 \%$ placed the rest on the mesial while $18.8 \%$ placed the rest on the distal aspect of the abutment tooth. The concern here is $12.5 \%$ (Fig. 5) of the designs did not have provision for rest placement and these cases were observed when 8 or more teeth needed to be replaced. Where a denture relied heavily on tissue or mucosal support, this would create a situation where there would be concentration of load on 
the mucoperiosteum. This is likely to accelerate bone resorption and allow the saddle to sink in and occlusion will be affected. Broad coverage from the saddle area and smaller occlusal table would be recommended in this case to reduce the load on the ridge (18).

The type of clasp, material used and position of retentive arm of clasps on abutment teeth were analyzed. The use of gingivally approaching clasps were observed in $30 \%$ of the designs in the lower casts (Figure 6a). Half of these cases were free end saddle situation. The concept of utilising a longer clasp arm of at least 15 $\mathrm{mm}$ in the form of gingival approaching clasp or a platinised gold or stainless steel alloy which has a higher elastic limit was advised by Bates (19-20). This length of $15 \mathrm{~mm}$ or less is impossible to be obtained when occlusal approaching clasp is used on premolars. The concern here is that, $64 \%$ of the premolar abutments were designed with occlusal approaching clasp (Fig 6b). This clasp when used on the tooth, would give considerable leverage to the abutment tooth and stress the periodontal membrane. The use of gingival approaching clasp may protect the premolar abutment from this damaging stress (17-18).

The choice of connectors for lower designs were analysed and presented in Table 4 . Only 2 types of major connectors were observed in this survey i.e the lingual bar and palatal plate. The lingual bar was mostly used with 13 out of 16 cases $(81.7 \%)$. The popularity of the lingual bar was also noted in the work by Basker et al (12).

Clinically, the use for lingual bars is restricted by the anatomical situation. The amount of space available for lingual bar placement may be determined during the clinical examination and this may be confirmed in the master cast.

The lingual plate was used in all cases where 6 or more teeth were to be replaced and this indicate an understanding in ensuring adequate major connector rigidity, which will provide better support and load distribution. (Table 4)

When designing removable partial denture, it has been recommended that gingival margins should remain uncovered and that the distance from the gingival margin to the denture base should be at least $3 \mathrm{~mm}$. This is to avoid trauma from the denture base (21-23).

The decision to restrict gingival coverage in situations where 'replacement of 6 or less teeth' was based on the work of Basker and Davenport (10). They felt that it would be impractical to uncover gingival margins in situations where there are more than 6 missing teeth in the posterior. The results of the present survey indicated that there were 62 cases where there were 6 or less teeth which needed to be replaced. Of this, only $29 \%$ had gingival margins covered. It is good to note that the principle of minimal coverage was followed.

Although the information and data collected in this study is restricted due to time constraint and sample size, it is useful as a reference for further studies. For future studies, it is suggested that a bigger sample size covering other states in Malaysia is used. It is also recommended that the study be carried out for a longer duration.
It would be interesting to include a section in the survey on why dentists relied solely on the technician to design the dentures.

The outcome of the survey indicated that half of the dentist gave either detailed or guided instruction on partial denture design to the technicians. This shows that there is some form of communication between the clinician and technician.

A periodic continuing dental education on denture designing and related topics for dental graduates and dental technicians would be useful to improve communication between the clinician and technician. Trainor et al (24) recommended that these refresher courses provide reinforcement in the basic design concept and help dentists to gain confidence in partial denture designing.

\section{CONCLUSIONS}

One hundred and ten frameworks from three dental laboratories in Kuala Lumpur and Shah Alam were examined. There were more upper casts sent for framework fabrication compared to the lower. The results of this study indicated that $43.6 \%$ of the dentist did not provide proper partial denture designs to the technician. About $6.4 \%$ of the dentist had requested that the technicians design the framework. Only $18.2 \%$ of the dentists gave detailed instruction on the type of components to be used in the denture design.

There were different variations in designs. On the whole these designs, were considered acceptable with regard to the biologic and mechanical requirements for a cast cobalt-chromium partial denture.

It is suggested that continuing dental education involving both the dentist and dental technician be routinely conducted on partial denture design. It is hoped that this will provide reinforcement in the knowledge of the basic removable partial denture design concept and also improve communication between dentists and dental technicians.

\section{ACKNOWLEDGEMENTS}

This paper was part of a poster presentation at the $2^{\text {nd }}$ Dental Student Scientific Conference held in Perdanasiswa, University of Malaya on the 10-11 Nov. 2000. The authors wished to thank

1) The three commercial dental laboratories involved for their co operation and contribution to all the datas:

a) Asia Dental Lab., Jalan Klang Lama, KL.

b) Cosmo-Dent Lab., Shah Alam.

c) Mega Chrome Lab., Jalan Klang Lama, KL.

2) Faculty of Dentistry, University of Malaya for the funding.

3) Mohd. Ismail (Dental year V undergraduate) for his technical support. 


\section{REFERENCES}

1. Academy of Prosthodontics. J Prosthet Dent 1995; 73: 81-84.

2. McCracken W L. Survey of partial denture designs by commercial dental laboratories. J Prosthet Dent 1962; 12: 1089 .

3. Henderson D. Writing work authorization for removable partial dentures. J Prosthet Dent 1966; 16: 696-707.

4. Sykora O, Calikkocoaglu S. Maxillary removable partial denture designs by commercial dental laboratories. J Prosthet Dent 1970; 23: 633.

5. Owall B. Design of removable partial dentures and dental technician education. Swed Dent J 1974; 67: 21.

6. Von Steyern P.Vult, Widolf-Kroon R, Nilner K, Basker R M. Removable partial denture design habits in general dental practice in Sweden. Swed Dent J 1995; 19: 205-211.

7. Schwarz W D, Barsby M J. Design of partial denture in dental practice. J Dent 1978; 6: 166-170.

8. Stafford G D, Glantz P-O, Harrison A, Murphy W M. A comparison of some aspects of dental technology in commercial laboratories in England and Sweden. Swed Dent J 1982; 6: 81-86.

9. Hardy F, Stuart L M. A critique of materials submitted by dentists to dental laboratories for the fabrication of removable partial dentures. Quintessence Dent Technol 1983; 7: 93-95.

10. Basker R M, Davenport J C. A survey of partial denture design in general dental practice. $\mathrm{J}$ Rehab 1978; 5 : 215-222

11. Sykora O. Removable partial denture design by Canadian dental laboratories: A retrospective Study. J Can Dent Assoc 1995; 61: 615-612.

12. Basker R M,Davenport J C, Harrison A, Marshall $\mathrm{J}$ L. Partial denture designs in general dental practice-10 years on. Brit Dent J 1988; 165: 245249.
13. Taylor T D, Matthews A C, Aquilino S A et al. Prosthodontic survey. Part II : Removable prosthodontic curriculum survey. J Prosthet Dent 1984; 52 : 747-749.

14. Holt R D, Rule D C and Basker R M et al. The influence on partial denture design of a teaching video for general dental practitioners. Br Dent J 1994; 176: 379-385.

15. Kennedy E. Partial denture construction. Dental Items of Interest 1923; 47: 23.

16. Barsby MJ. Partial denture design. Part 2. Basic Principles of design. Dental Update 1979: 9-21

17. Kratochvil F J. The influence of occlusal rest position and clasp design on movement of abutment teeth. J Prosthet Dent 1963; 13: 114-123.

18. Ritchie GM Partial Denture Design. Lower free-end saddle dentures. Dental Update 1982: 429-442.

19. Bates J F (1956) Proc. Brit. Soc.Study Pros. Dent. P. 17

20. Bates J F. The mechanical properties of the cobaltchromium alloys and their relation to partial denture design. Br Dent J 1965; 119: 389-396.

21. Storer R. Gingival care in Prosthetic Dentistry. Dental Practitioner and Dental Records. 1969; 19: 187.

22. Derry A and Bertram U. A clinical survey of removable partial dentures after 2 years of usage. Acta Odontologica Scand 1970; 28: 581.

23. Bissada NF, Ibrahim SI, Barsoum WM. Gingival response to various types of removable partial dentures. J Periodontol 1974; 45: 651-659.

24. Trainor J E, Elliot R W Jr and Bartlett S D. Removable partial dentures designed by dentist before and after undergraduate level : A comparative study. J Prosthet Dent 1972; 5: 509. 